\title{
Search for GeV neutrinos associated with solar flares with IceCube
}

\author{
The IceCube Collaboration ${ }^{\dagger}$ \\ $\dagger$ http://icecube.wisc.edu/collaboration/authors/icrc17_icecube \\ E-mail: gdewassedicecube.wisc.edu
}

\begin{abstract}
Since the end of the eighties and in response to a reported increase in the total neutrino flux in the Homestake experiment in coincidence with solar flares, solar neutrino detectors have searched for solar flare signals. Hadronic acceleration in the magnetic structures of such flares leads to meson production in the solar atmosphere. These mesons subsequently decay, resulting in gamma-rays and neutrinos of $\mathrm{O}(\mathrm{MeV}-\mathrm{GeV})$ energies. The study of such neutrinos, combined with existing gamma-ray observations, would provide a novel window to the underlying physics of the acceleration process. The IceCube Neutrino Observatory may be sensitive to solar flare neutrinos and therefore provides a possibility to measure the signal or establish more stringent upper limits on the solar flare neutrino flux. We present an original search dedicated to low energy neutrinos coming from transient events. Combining a time profile analysis and an optimized selection of solar flare events, this research represents a new approach allowing to strongly lower the energy threshold of IceCube, which is initially foreseen to detect $\mathrm{TeV}$ neutrinos.
\end{abstract}

Corresponding author: G. de Wasseige*

IIHE-VUB, Pleinlaan 2, 1050 Brussels, Belgium

35th International Cosmic Ray Conference

10-20 July, 2017

Bexco, Busan, Korea

* Speaker. 


\section{Introduction}

In 1988, the Homestake experiment reported an increase in the total number of neutrino events, potentially correlated with energetic solar flares [1]. J.N. Bahcall predicted that if this increase were indeed due to solar flares, it would lead to large characteristic signals in neutrino detectors [2]. In response, experiments such as Kamiokande [3] and SNO [4] performed several studies. Using different solar flare samples and analyses, the experiments were not able to confirm the potential signal seen by Homestake.

Solar flares convert magnetic energy into plasma heating and kinetic energy of charged particles such as protons [5]. Injected downwards from the coronal acceleration region, protons can interact with the dense plasma in the lower solar atmosphere through the processes indicated in Eq. 1.1 where the energy thresholds are $280 \mathrm{MeV}$ and $180 \mathrm{MeV}$ for $\mathrm{p}-\mathrm{p}$ and $\mathrm{p}-\alpha$ respectively.

$$
p+p \text { or } p+\alpha \longrightarrow \begin{cases}\pi^{+}+X ; & \pi^{+} \longrightarrow \mu^{+}+v_{\mu} ; \mu^{+} \longrightarrow e^{+}+v_{e}+\bar{v}_{\mu} \\ \pi^{0}+X ; & \pi^{0} \longrightarrow 2 \gamma \\ \pi^{-}+X ; & \pi^{-} \longrightarrow \mu^{-}+\bar{v}_{\mu} ; \mu^{-} \longrightarrow e^{-}+\bar{v}_{e}+v_{\mu}\end{cases}
$$

Due to their hadronic origin, solar flare neutrinos are of great interest for investigating proton acceleration and the subsequent interactions in the chromosphere. As demonstrated in several studies (see e.g. $[6,7,8]$ ), this neutrino flux extends from $\mathrm{MeV}$ up to a few $\mathrm{GeV}$. Neutrino facilities sensitive to this energy regime could open a new window on solar flare physics and provide new constraints on e.g. the proton spectral index, as it is shown in these proceedings. In the following, we will focus on the IceCube Neutrino Telescope and its sensitivity to the high energy part of the neutrino spectrum from solar flares. Section 2 introduces a new event selection lowering the threshold of IceCube down to the GeV level. Combined with a dedicated time profile analysis described in Section 4, this selection allows IceCube to be sensitive to astrophysical transient events such as solar flares. The potential physics reach when applying this combination is described at the end of Section 4.

\section{The IceCube Neutrino Observatory: from TeV to $\mathrm{GeV}$}

The IceCube Neutrino Observatory is a cubic-kilometer neutrino detector installed in the ice at the geographic South Pole between depths of $1450 \mathrm{~m}$ and $2450 \mathrm{~m}$, completed in 2010 [9, 10]. A lower energy infill, the DeepCore subarray, includes 8 densely instrumented strings with smaller spacing between its optical modules ( $7 \mathrm{~m}$ versus $17 \mathrm{~m}$ in the IceCube strings) and its strings (72 $\mathrm{m}$ in average versus $125 \mathrm{~m}$ in IceCube) [11]. When a neutrino interacts in the neighborhood of the detector, the subsequent electromagnetic and/or hadronic cascade emit Cherenkov photons that can be detected by one or several of the 5160 optical modules distributed over the $1 \mathrm{~km}^{3}$ volume.

Originally dedicated to observe $\mathrm{TeV}$ neutrinos, IceCube has demonstrated the ability to extend its sensitivity to a larger energy range by the use of DeepCore. Indeed, since the observation of the first astrophysical neutrinos in 2013 [12], the IceCube collaboration has set several noteworthy limits on, among others, the existence of sterile neutrinos [13] and the spin-dependent WIMP-nucleon cross section [15] as well as competitive measurements of neutrino oscillation parameters [14]. The 


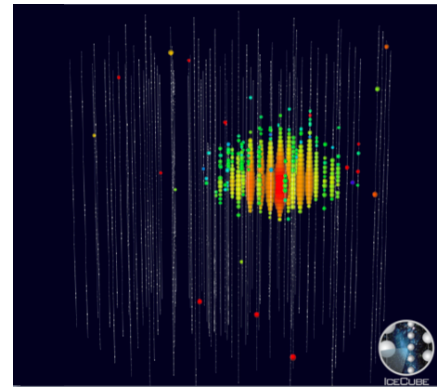

(a) PeV neutrino interaction

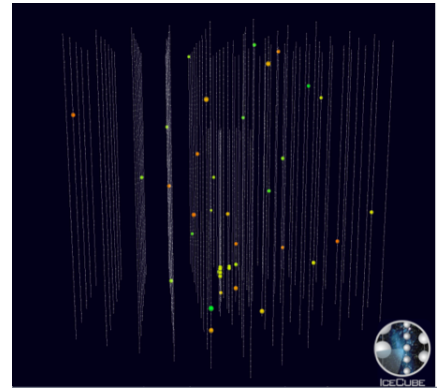

(b) $\mathrm{GeV}$ neutrino interaction

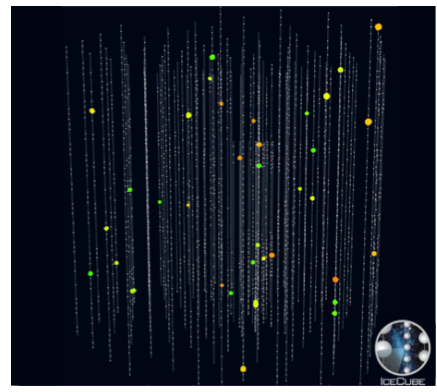

(c) Detector noise event

Figure 1: Examples of neutrino interactions as seen in IceCube. A typical $\mathrm{GeV}$ neutrino interaction is illustrated in $1 \mathrm{~b}$ while $1 \mathrm{a}$ and $1 \mathrm{c}$, respectively, show the well-known "Ernie" event in the PeV range and a detector-noise event (see the text for more details).

collaboration has also joined the worldwide multimessenger effort studying the highest energetic events in our Universe [16, 17, 18].

The work presented in these proceedings will demonstrate a new selection capable of extending the sensitivity to neutrino energies around $1 \mathrm{GeV}$ as well as the use of external facilities/experiments to define a time window of interest. With these two tools, IceCube will be able to study astrophysical transient events down to $\mathrm{GeV}$ scales.

\section{Selection of GeV events}

The event selection presented here is optimized for neutrino interactions happening in the DeepCore volume. A softer trigger condition is implemented in this subdetector in order to increase the sensitivity to lower neutrino energies. Three hit optical modules satisfying a Hard Local Coincidence (HLC) ${ }^{1}$ within a $2.5 \mu$ s time window are required to define an "event". Furthermore, the sample only contains events that did not fulfill the conditions to pass the running filters [10], except for the DeepCore Filter and either of two additional filters dedicated to low-energy neutrinos: the Low-up filter (LowUp) and/or the Full-Sky-Starting filter (FSS). The LowUp filter has been designed to target low-energy neutrinos coming from the Northern sky while the FSS filter uses parts of the detector as veto for incoming muon events, allowing to search for low-energy neutrinos from all directions. This results in a significant reduction of the number of atmospheric muons from the original rate of $1400 \mathrm{~Hz}$ to $15 \mathrm{~Hz}$ - while retaining more than $98 \%$ of signal events.

As illustrated in Fig.1, the main difference between $\mathrm{TeV}$ and $\mathrm{GeV}$ neutrino interactions is the amount of light emitted in the ice. Putting strong constraints on the number of optical modules hit eliminates neutrinos and remaining muons with an energy exceeding $5 \mathrm{GeV}$. A distinction is made between IceCube strings (i.e. strings with a spacing of $125 \mathrm{~m}$ ) and DeepCore strings (i.e. with a $72 \mathrm{~m}$ average spacing) to optimize the selection of DeepCore events. An upper limit on the number of causally connected optical modules, i.e. the DOMs that have likely observed the same physics interaction, further helps to select low energy events. A GeV neutrino interaction produces a small cascade or a short track emitting light close to the interaction vertex, resulting in a small number

\footnotetext{
${ }^{1}$ At least two hit DOMs in a next- or next-to near neighbor vertical span in a time window of $\pm 1 \mu \mathrm{s}$.
} 
Table 1: Summary of the conditions required for an event to be selected as a GeV-like neutrino interaction.

\begin{tabular}{r|l}
\hline Variable & Passing condition \\
\hline Trigger N HLC-DOMS & $\geq 3$ \\
Passing filters & DeepCore \\
& or \\
& DeepCore $+($ Low Up and/or $F S S)$ \\
\hline $\mathrm{N}_{\text {HLC-DOMS in IceCube strings }}$ & $\leq 6$ \\
$\mathrm{~N}_{\text {HLC-DOMS in DeepCore strings }}$ & $\leq 7$ \\
$\mathrm{~N}_{\text {DOMS }}$ causally connected & $\leq 10$ \\
\hline
\end{tabular}

of causally connected DOMs. A summary of the conditions required for an event to be selected as a GeV-like neutrino interaction is listed in Table 1.

\subsection{Minimizing the contribution of pure noise events}

Besides high energy muons and neutrinos that could still contaminate the low energy event sample, pure detector noise events may trigger DeepCore and pass the above selection. A detailed simulation of noise in the detector helps to estimate the potential contamination of accidental triggers caused by detector noise, hereafter referred to as "noise events". These include uncorrelated thermal noise, uncorrelated radioactive noise, and correlated scintillation noise [19]. About $6 \mathrm{~Hz}$ of pure noise survives the selection described above, being the dominant contribution of the event sample. It is therefore important to minimize this contribution.

Events which have hits that are causally connected are kept, while events containing many non-causally connected hits are removed. The causality between two hits is stated if their effective speed - i.e. the distance between the two hits divided by the time separation between them - is consistent with the speed of light in ice. Applying the causality condition significantly reduces the rate of detector noise events, from $15 \mathrm{~Hz}$ to $0.2 \mathrm{~Hz}$.

\subsection{Rate and detector stability}

After the event selection described above, the data rate is around $0.2 \mathrm{~Hz}$ while more than $50 \%$ of the neutrino interactions below $5 \mathrm{GeV}$ generated with Genie [20] with a generic $\mathrm{E}^{-2}$ spectrum pass the selection.

Even if this rate is still substantially larger than the expectation of atmospheric neutrinos, estimated to occur at the $\mathrm{mHz}$ level, the selection is sensitive both to single transient events as for an event-stacking analysis. The sensitivity depends on the stability of the detector once the event selection is applied. Fig. 2 shows the rate, smoothed with a low pass filter, for three periods of time occurring in 2011-2013. Even though Poissonian fluctuations are present, the overall behavior of the rate is constant along the seasons and the years.

The actual sensitivity of this event selection to astrophysical transient events depends on the characteristics of the event class itself. In the next section, we focus on solar flares, defining a specific framework that allows IceCube to be sensitive in the GeV energy range. 


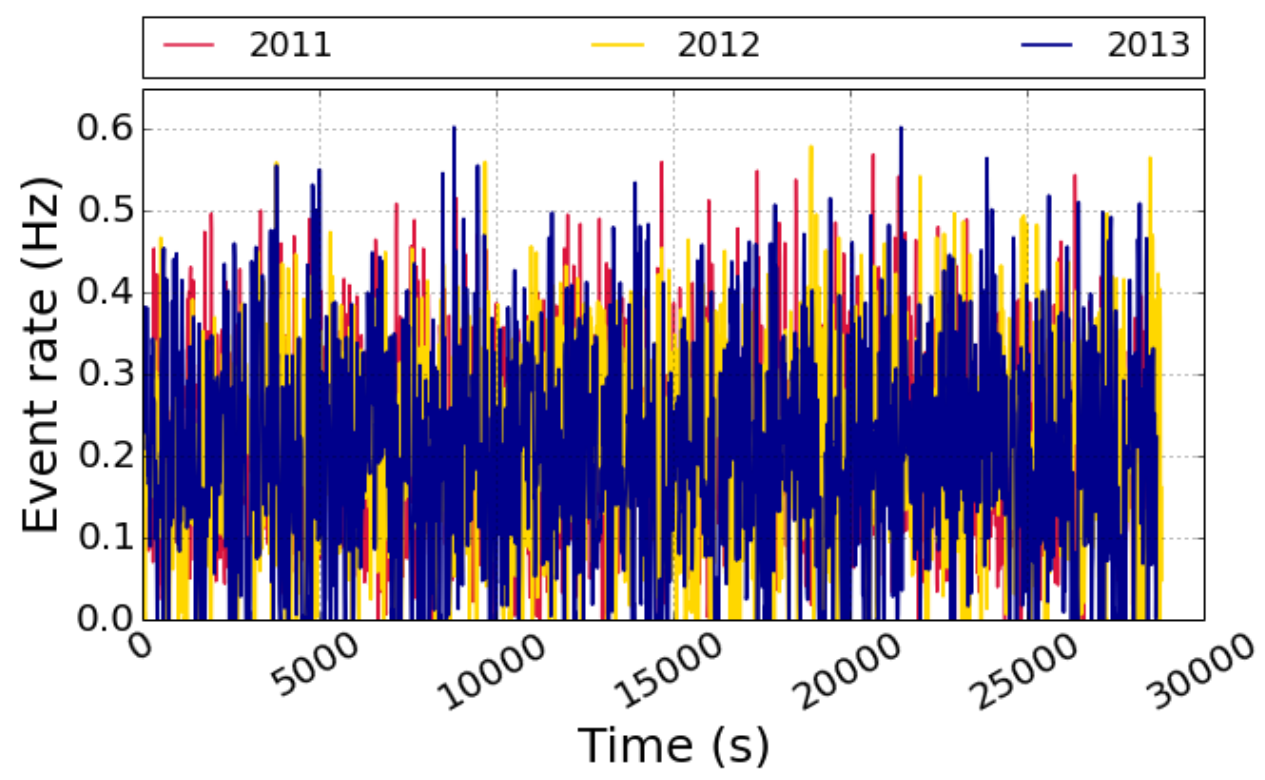

Figure 2: Detector rate using a $10 \mathrm{~s}$ smoothing and an order 3 low-pass butterworth filter. Three different periods of the year in 2011 (red), 2012 (yellow) and 2013 (blue) are shown. To prevent unblinding issues with future transient searches using the same event selection, the absolute time of the data has been omitted. Even though there are Poissonian fluctuations, the mean values, $0.22 \mathrm{~Hz}(2011), 0.20 \mathrm{~Hz}$ (2012) and $0.20 \mathrm{~Hz}$ (2013), are consistent with each other.

\section{Solar flare application}

The work presented in these proceedings uses the event selection described above to search for neutrinos from solar flares. As described in [21], one can select solar flares with high probability of pion production in order to optimize this search for neutrinos. Due to their common production channel through the decay of pions, gamma-rays and neutrinos are expected to be emitted simultaneously. Therefore, using observations from gamma-ray detectors such as Fermi-LAT [22] allows one to define solar flare events of interest as well as precise time windows for an optimal neutrino search.

It is also interesting to note that Fermi-LAT has been able to detect the impulsive phase in some of the latest solar flare events. These impulsive phases are characterized by a small full-width at half-maximum, narrowing down the neutrino search window to a few minutes. Moreover, the analysis of the gamma rays detected during these short phases reveals a relatively hard initial proton spectrum, with a spectral index around 3, as well as an enhanced gamma ray yield [23]. The long duration emissions, on the contrary, show a softer proton spectral index (between 4 and 6) and a spread of the gamma-ray emission over several hours. Focusing on the impulsive phase of bright events of the last solar cycle therefore increases the chance of a neutrino detection in coincidence with solar flares.

These proceedings focus on an observed event on March 7th, 2012, for which the Fermi-LAT instrument was able to detect both the impulsive and long duration emissions [23]. Furthermore, the Fermi collaboration could realize a spectral analysis of the impulsive phase. One can then 


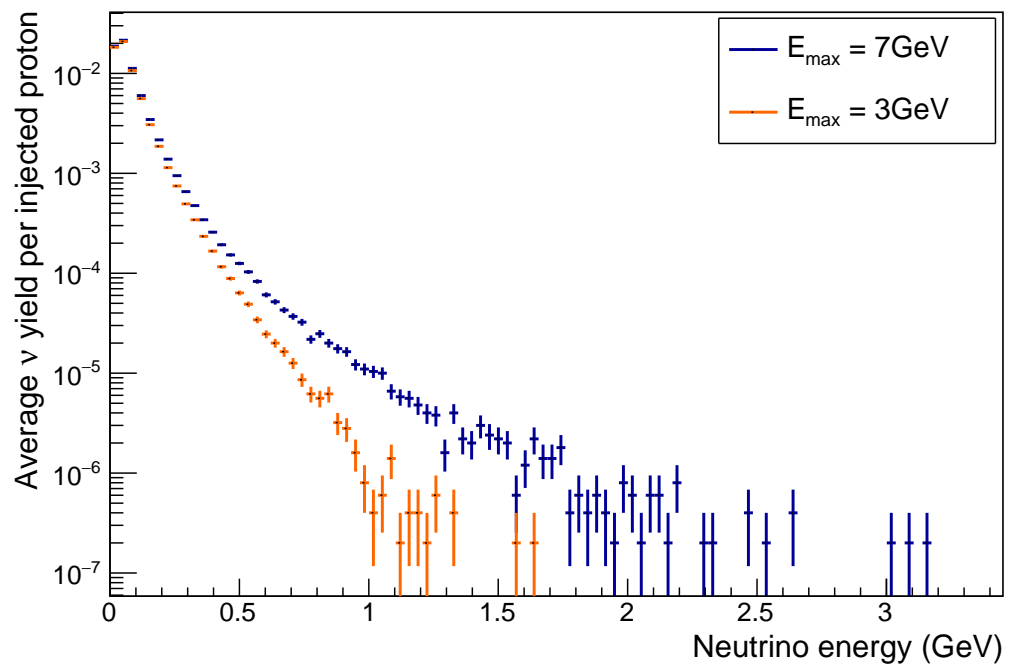

Figure 3: Average neutrino yield per injected proton with a spectral index of 3.2 and a upper cutoff of $7 \mathrm{GeV}$ (blue) and $3 \mathrm{GeV}$ (orange). A higher cutoff value leads to a higher neutrino yield in the energy range targeted by the event selection presented in this work.

define the optimal time window using gamma-rays with an energy greater than $500 \mathrm{MeV}$, focusing in this way on the energy range where the selection defined above is sensitive. In the following, the first 20 minutes of the March 7th, 2012 event as detected by Fermi-LAT will be considered.

Following the method described in [6, 24], using the proton spectral index extracted from gamma ray observations by Fermi-LAT [23] for this particular event, one can estimate the corresponding numbers of neutrino interactions detectable by IceCube. A potential upper cutoff of the proton spectrum has been discussed in several studies (see e.g. [25]). While an exponentially falling spectrum could be present after this cutoff, we use here a Heaviside step function in order to be conservative on the expected neutrino flux. The proton flux is therefore defined as $\frac{d \phi}{d E}=A E^{-\delta} H\left(E_{\max }-E\right)$, where $\mathrm{A}$ is a normalisation constant, $\delta$ represents the spectral index and $\mathrm{E}_{\max }$ is the upper cutoff. The effect of this upper cutoff on the subsequent neutrino flux is illustrated in Fig. 3, where the blue (orange) points show the average neutrino yield per injected proton with $\delta=3.2$ and $E_{\max }=7 \mathrm{GeV}(3 \mathrm{GeV})$. A higher cutoff value leads to a higher neutrino yield in the energy range targeted by the event selection described in Section 3.

Table 2 shows the expected number of events passing the event selection described in Section 2 for different upper cutoff values. Fig. 4 illustrates these numbers together with the number of events required for a 1-, 3-, and 5-sigma deviation from the background expectation in a counting experiment. IceCube has the potential to find a signal or constrain this upper cutoff using the strength of the signal detected during this solar flare. The same procedure can and will be repeated for the brightest solar flare events from the 24th solar cycle listed in [26, 21].

\section{Summary}

These proceedings describe a new event selection that allows the IceCube telescope to be 
Table 2: Expected number of solar flare neutrino events passing the selection described in Section 2 for different values of the upper cutoff. These numbers have been obtained assuming a proton spectral index of 3.2, averaged and derived from observations of the March 7th, 2012 event [23]. For comparison, the last line shows the background expectation.

\begin{tabular}{c|c}
\hline Value of the upper cutoff & Expected number of events in IceCube \\
\hline $3 \mathrm{GeV}$ & $19.5 \pm 0.3+\mathrm{Bkg}$ \\
$5 \mathrm{GeV}$ & $43.2 \pm 0.4+\mathrm{Bkg}$ \\
$7 \mathrm{GeV}$ & $94.3 \pm 0.7+\mathrm{Bkg}$ \\
\hline Bkg = No signal (2011) & $264 \pm 16$ \\
\hline
\end{tabular}

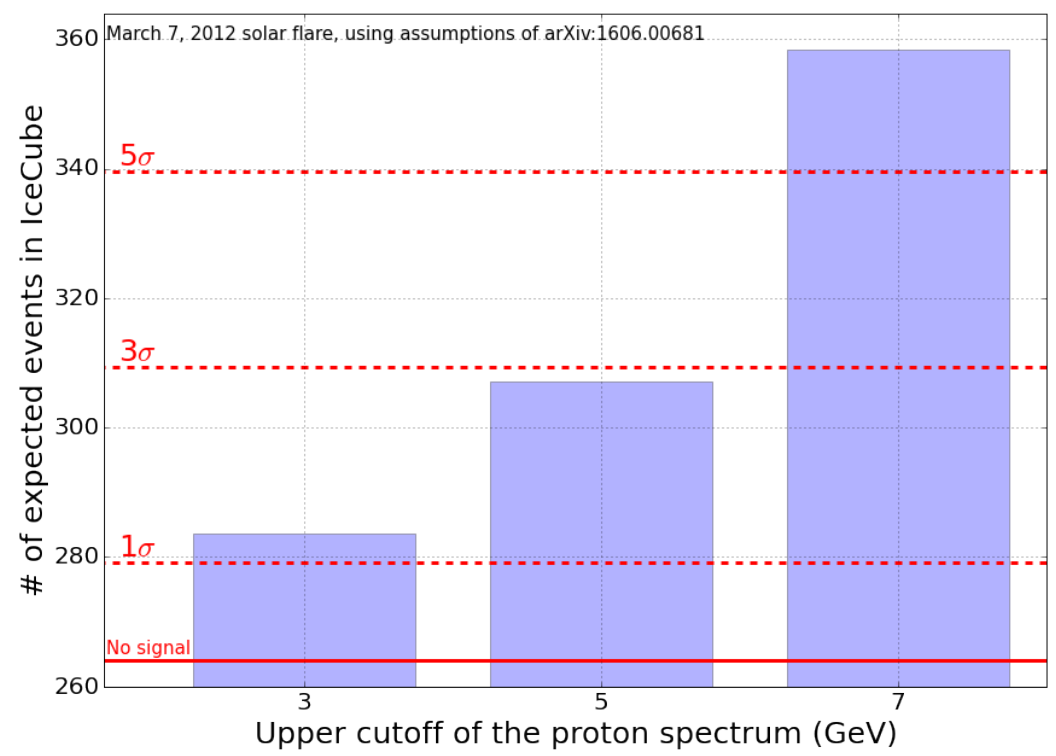

Figure 4: Comparison of the expected number of events in IceCube for the solar flare of March 7th, 2012 and the required number of events for a 1-, 3-, and 5-sigma deviation from background expectation in a counting experiment.

sensitive to $\mathrm{GeV}$ neutrinos, and therefore perform low-energy searches for astrophysical transient events, such as solar flares or gamma-ray bursts. This sensitivity to low energy neutrinos can be achieved by the use of electromagnetic observations to define the optimal time window for neutrino searches. An application to solar flare events, and especially to the bright event of March 7th, 2012, was presented. In the framework of solar flares, the Fermi-LAT instrument can define the events of interest as well as the optimal time window for a neutrino search. Assuming the proton spectral index derived from the Fermi-LAT observations, the number of events expected to pass the above selection has been established as a function of the upper cutoff of the proton spectrum. As illustrated in Fig. 4, IceCube has e.g. the potential to constrain this upper cutoff using the strength of the observed signal. 


\section{References}

[1] R. Davis, Nucl. Phys. B 48 (1996) 284.

[2] J.N. Bahcall, Phys. Rev. Lett. 61 (1988) 2650.

[3] K.S. Hirata et al., Phys. Rev. Lett. 61 (1988) 2653.

[4] B. Aharmim et al., Astropart. Phys. 55 (2014) 1.

[5] H.S. Hudson, Space Sci. Rev. 158 (2011) 5.

[6] G. de Wasseige for the IceCube Collaboration, Moriond EW (2016). arXiv:1606.00681 [astro-ph.HE].

[7] D. Fargion, JHEP 0406 (2004) 045.

[8] G.E. Kocharov et al., Il Nuevo Cimento 14C (1991) 4.

[9] IceCube Collaboration, A. Achterberg et al., Astropart.Phys. 26 (2006) 155.

[10] IceCube Collaboration, M. G. Aartsen et al. JINST 12 (2017), P03012.

[11] IceCube Collaboration, R. Abbasi et al., Astropart. Phys. 35 (2012) 615.

[12] IceCube Collaboration, M. G. Aartsen et al. Science 342 (2013) 1242856.

[13] IceCube Collaboration, M. G. Aartsen et al., Phys. Rev. Lett. 117 (2016), 071801.

[14] IceCube Collaboration,M. G. Aartsen et al., Nucl. Phys. B 908 (2016) 161.

[15] IceCube Collaboration, M. G. Aartsen et al., Eur. Phys. J. C 77 (2017), 146.

[16] M. W. E. Smith et al., Astropart. Phys. 45 (2013) 56.

[17] IceCube Collaboration, M. G. Aartsen et al., Astropart. Phys. 92 (2017) 30.

[18] IceCube Collaboration, PoS ( ICRC2017) 1007 (these proceedings).

[19] M. Larson, Ph.D thesis, University of Alabama, Tuscaloosa (2013).

[20] C. Andreopoulos, Acta Phys. Polon. B 40 (2009) 2461.

[21] G. de Wasseige et al., (2015) arXiv:1505.05837 [astro-ph.HE].

[22] W. B. Atwood et al., Astrophys. J. 697 (2009) 1071.

[23] M. Ajello et al., Astrophys. Journ. 789 (2014) 20.

[24] G. de Wasseige et al., POS (ICRC2015) 1049 (2016).

[25] Dj. Heristchi et al., Sol.Phys. 49 (1976) 151.

[26] M. Ackermann et al., Astrophys. Journ. 787 (2014) 15. 